\title{
An Analytical Study for Caputo Fractional Derivative on Unsteady Casson Fluid with Thermal Radiation Effect
}

\author{
Ridhwan Reyaz, Ahmad Qushairi Mohamad*, Yeaou Jiann Lim, Muhammad Saqib, \\ Zaiton Mat Isa, Sharidan Shafie
}

Department of Mathematical Sciences, Faculty of Science, Universiti Teknologi Malaysia, 81310 Johor Bahru, Johor, Malaysia

Received August 20, 2021; Revised September 12, 2021; Accepted October 17, 2021

\section{Cite This Paper in the following Citation Styles}

(a): [1] Ridhwan Reyaz, Ahmad Qushairi Mohamad, Yeaou Jiann Lim, Muhammad Saqib, Zaiton Mat Isa, Sharidan Shafie, "An Analytical Study for Caputo Fractional Derivative on Unsteady Casson Fluid with Thermal Radiation Effect," Mathematics and Statistics, Vol. 9, No. 6, pp. 867 - 881, 2021. DOI: 10.13189/ms.2021.090602.

(b): Ridhwan Reyaz, Ahmad Qushairi Mohamad, Yeaou Jiann Lim, Muhammad Saqib, Zaiton Mat Isa, Sharidan Shafie (2021). An Analytical Study for Caputo Fractional Derivative on Unsteady Casson Fluid with Thermal Radiation Effect. Mathematics and Statistics, 9(6), 867 - 881. DOI: 10.13189/ms.2021.090602.

Copyright $\odot 2021$ by authors, all rights reserved. Authors agree that this article remains permanently open access under the terms of the Creative Commons Attribution License 4.0 International License

\begin{abstract}
Studies on Casson fluid are essential in the development of the manufacturing and engineering fields since it is widely used there. Meanwhile, fractional derivative has been known to be a constructive paradox that can be beneficial in the future. In this study, the development fractional derivative on Casson fluid flow is investigated. A fractional Casson fluid model with effect of thermal radiation is derived together with momentum and energy equations. The Caputo definition of fractional derivative is used in the mathematical formulation. Casson fluid with constant wall temperature over an oscillating plate in the presence of thermal radiation is considered. Solutions were obtained by using Laplace transform and are presented in the form of Wright function. Graphical analysis on velocity and temperature profiles was conducted with variations in parametric values such as fractional parameter, Grashof number, Prandtl number and radiation parameter. Numerical computations were carried out to investigate behaviours of skin friction and Nusselt number. It is found that when the fractional parameter is increased, the velocity and temperature profiles will also increase. Existence of fractional parameter in both velocity and temperature profiles shows the transitional phenomenon of both profiles from an unsteady state to steady state, providing a new perspective on Casson fluid flow. An increment in both profiles is also observed when the thermal radiation parameter is increased. The present
\end{abstract}

results are also validated with published results, and it is found that they are in agreement with each other.

Keywords Casson Fluid, Caputo Derivative, Thermal Radiation, Wright Function

\section{Introduction}

The concept of fractional calculus first surfaced when L'Hospital wrote a letter to Leibniz, who invented the $x$ notation, asking the outcome if $n$ is $1 / 2$ [1]. Since then, Bernoulli [2], Wallis [3], Euler [4], Laplace [5], Lacroix [6] and Fourier [7] have mentioned fractional calculus in their writings. Riemann, Heaviside, Abel and Liouville developed the fundamental applications and theory of fractional calculus [8-11]. Fractional has proven to be more accurate compared to conventional ordered derivative models [1213]. Debnath [14] wrote a review on applications of fractional calculus in fields of engineering and science. Some applications can be found in dynamical systems in control theory, viscoelasticity, electrochemistry and model of neurons in biology. Over time, many definitions of fractional integral and derivative have been developed and applied. Dalir [15] and De Oliveira [16] did an extensive review on the applications of different definitions of fractional calculus in different fields of 
engineering and science. Caputo derivative is one of the many definitions for fractional derivatives. Solving a fractional model with the Caputo derivative will result in a special Wright function. The Wright function was first introduced by Wright in the 1940s [17-18]. It has been widely used to solve fractional ordered partial difference problem.

The non-Newtonian fluid is a complicated fluid to comprehend. Due to its physical properties, researchers are having a challenge for solving non-Newtonian fluid problems. There is yet a comprehensive model that has all the properties of a non-Newtonian fluid. Nevertheless, since the fluid has gain popularity in the manufacturing and processing industry, scientists are still trying to develop new models until now. One such model would be the Casson fluid model. It was introduced by Casson [19] in 1959 for a rheological data of pigment oil suspensions in printing ink. A Casson fluid is defined as a shear-thinning liquid that has infinite viscosity at zero rates of shear [20]. To date, the Casson model is considered to be one of the best rheological models. In comparison, a viscoplastic model would be less accurate at high and low shear rates. Not only that, Casson fluid is one of the least complex non-Newtonian fluid model to be solved analytically. The fact that analytical studies on fractional derivatives are relatively new, a simple model is used to avoid unnecessary complication while calculating the final solutions. Past literature on fractional Casson fluid includes Khalid et al. [21], Khan et al. [22], Ali et al. [12] and Saqib et al. [23]. Studies have shown, analytical solutions from fractional models are significantly different from the classical models, providing an advantage to some practical problem [24].

By considering a free convection flow of Casson fluid in a porous medium, Khalid et al. [21] obtained the exact solutions using Laplace transform. Khan et al. [22] extended the study by introducing fractional calculus to the generalised Casson fluid and considering oscillating boundary conditions. Again, the solution was obtained through the Laplace transform. Later, Ali et al. [12] modified the model to consider a generalised Casson fluid with an infinite plate instead of a finite plate. Lastly, Saqib et al. [23] also modified the model by introducing the presence of first-order chemical reaction and considering slip effect.

Thermal radiation is defined as electromagnetic radiation generated from thermal motion of particles in matter. Fluid flow with presence of thermal radiation can be observed in many engineering and manufacturing process. Some of these include nuclear power generators, jet engines, turbines and many more. Over the years, investigations radiative heat on boundary fluid flow includes Prasad et al. [25] investigated the effects of thermal radiation on an MHD free convective heat and mass transfer flow of a nanofluid bounded by a semi-infinite flat plate. Biswas et al. [26] also did a study investigating the effects of thermal radiation on unsteady Casson fluid flowing vertically. Meanwhile, Raju et al. [27] studied the effects of thermal radiation on an unsteady MHD conducting fluid over an exponentially accelerated plate. Other studies on thermal radiation effects on fluid flow include Reddy et al. [28], Mohan [29] and Ibrahim et al. [30]. Effects thermal radiation on fluid flow with a fractional Casson fluid model have not been done.

To the best of authors' knowledge, an investigation on analytical study of Caputo fractional derivative for unsteady Casson fluid flow with thermal radiation effect has not been done. Therefore, this study aims to extend the work of Ali et al. [12] by considering thermal radiation effect in the generalised Casson fluid model with an oscillating plate in the presence of heat. The fractional model will be derived based on the Caputo derivative definition and solved with a Laplace transform. Solutions will be presented in terms of Wright function. Effects of a fractional parameter, thermal radiation and various other parameters will be presented numerically and graphically. Finally, results are also validated with Ali et al. [12].

\section{Problem Definition}

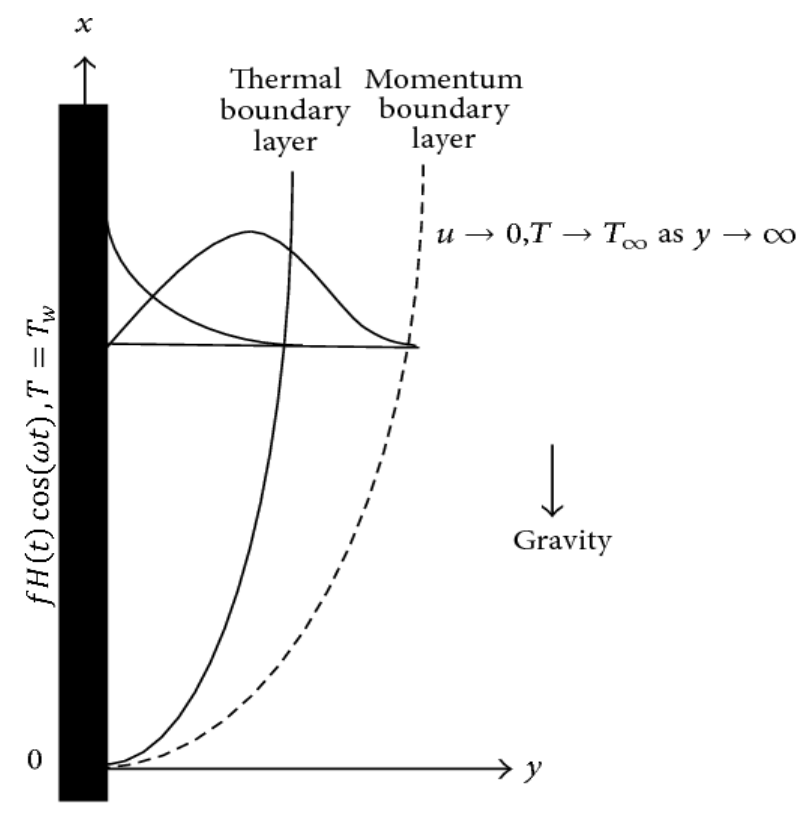

Figure 1. Physical representation

Incompressible Casson fluid with an unsteady convectional flow over an infinite flat vertical plate. $y$ is the coordinate measuring normal of the direction of the plate. The flow is constricted to 0 . The plate and the fluid is at rest at with constant temperature $T_{\infty}$. As time progresses, 0 , the oscillation of plate begins with velocity $f H(t) \cos (\omega t) . f$ is the amplitude, while $\omega$ is the frequency of plate oscillations and $H(t)$ is the unit step function. Meanwhile, the temperature of the plate is increased to $T_{w}$ and remained constant after that. Radiation effect is also taken into account such that the radiative heat flux is applied perpendicularly to the plate. 
The temperature and velocity are dependent on variable $y$ and $t$. A physical representation of fluid flow is displayed in Figure 1. Utilising the Boussinesq's approximation and considering a one-dimensional and unidirectional flow, the momentum and energy equation acquired are as follows [24-26]:

$$
\begin{gathered}
\frac{\partial u(y, t)}{\partial t}=v\left(1+\frac{1}{\beta}\right) \frac{\partial^{2} u(y, t)}{\partial y^{2}}+g \beta_{T}\left(T-T_{\infty}\right), \\
\rho c_{p} \frac{\partial T(y, t)}{\partial t}=k \frac{\partial^{2} T(y, t)}{\partial y^{2}}-\frac{\partial q_{r}}{\partial y} .
\end{gathered}
$$

subjected to initial and boundary conditions as such

$$
\begin{array}{ll}
u(y, 0)=0, & T(y, 0)=T_{\infty}, \\
u(0, t)=f H(t) \cos (\omega t), & T(0, t)=T_{W}, \\
u(\infty, t) \rightarrow 0, & T(\infty, t) \rightarrow T_{\infty},
\end{array}
$$

where $u$ is velocity and is the temperature of the fluid.

is the parameter for Casson fluid, is kinematic viscosity, is gravitational acceleration, $\beta_{T}$ is the thermal expansion coefficient, is fluid deinsity, $c_{p}$ is the specific heat under constant pressure and is thermal conductivity.

is the radiative heat flux. Using Rosseland's approximation and differentiating $q_{r}$ in terms of $y$, equation (2) becomes [20], [27-30]:

$$
\rho c_{p} \frac{\partial T(y, t)}{\partial t}=k \frac{\partial^{2} T(y, t)}{\partial y^{2}}-\left(-\frac{16 \sigma T_{\infty}^{3}}{3 k^{*}}\right) \frac{\partial^{2} T}{\partial y^{2}},
$$

where $\sigma$ is the Stefan-Boltzman constant and $k^{*}$ is the mean absorption coefficient.

To derive a generalised Casson fluid model, equations (1) and (4) are multiplied by $\lambda=v / f^{2}$. Then, replace $\lambda$ and

$\partial t$ on the left hand side with $\lambda^{\alpha}$ and $D_{t}^{\alpha}$ respectively. Thus, the following fractional model is acquired:

$$
\begin{aligned}
& \lambda^{\alpha} D_{t}^{\alpha} u(y, t)=\lambda v\left(1+\frac{1}{\beta}\right) \frac{\partial^{2} u(y, t)}{\partial y^{2}}+\lambda g \beta_{T}\left(T-T_{\infty}\right), \\
& \lambda^{\alpha} D_{t}^{\alpha} T(y, t) \rho c_{p}=\lambda k \frac{\partial^{2} T(y, t)}{\partial y^{2}}+\lambda\left(\frac{16 \sigma T_{\infty}^{3}}{3 k^{*}}\right) \frac{\partial^{2} T}{\partial y^{2}},
\end{aligned}
$$

where

$$
\lambda^{\alpha} D_{t}^{\alpha} \varphi(y, t)=\left\{\begin{array}{cc}
\frac{1}{\Gamma(1-\alpha)} \int_{0}^{t} \frac{\varphi^{\prime}(y, t)}{(t-\tau)^{\alpha}} d \tau ; & 0<\alpha<1, \\
\frac{\partial \varphi(y, t)}{\partial t} ; & \alpha=1,
\end{array}\right.
$$

is the Caputo differential operator of order and $\Gamma($.$) is$ the Gamma function.

$$
\begin{gathered}
v=\frac{u}{f}, \quad \xi=\frac{y}{\gamma}, \quad \gamma=\frac{v}{f}, \\
\tau=\frac{t}{\gamma}, \quad \theta=\frac{T-T_{\infty}}{T_{w}-T_{\infty}},
\end{gathered}
$$

Incorporating the dimensionless variables displayed above into equations (5) and (6) will generate [31-34]:

$$
\begin{aligned}
& D_{\tau}^{\alpha} v=\frac{\partial^{2} v}{\partial \xi^{2}}\left(1+\frac{1}{\beta}\right)+G r \theta, \\
& \operatorname{PrD}_{\tau}^{\alpha} \theta=(1+N) \frac{\partial^{2} \theta}{\partial \xi^{2}},
\end{aligned}
$$

where number, $\lambda\left(T_{w}-T_{\infty}\right) / f$ is the thermal Grashof is the Prandtl number and

$k$ is the thermal radiation parameter. The dimensionless boundary conditions are as follows

$$
\begin{array}{ll}
v(\xi, 0)=0, & \theta(\xi, 0)=0, \\
v(0, \tau)=H(\tau) \cos (\omega t), & \theta(0, \tau)=1, \\
v(\infty, \tau) \rightarrow 0, & \theta(\infty, \tau) \rightarrow 0 .
\end{array}
$$

\section{Solutions of Problem}

Final analytical solutions are obtained by first applying Laplace transform on Equations (8) and (9), subjected to initial conditions (10) to obtain the ordinary differential equations. Therefore, the final solution of the transformed equations and boundary conditions are as follows:

$$
\begin{array}{r}
\bar{V}(\xi, q)=\frac{q}{q^{2}+w^{2}} \exp -\xi \sqrt{\beta_{0} q^{\alpha}} \\
+\frac{G r_{0}}{q^{\alpha+1}} \exp -\xi \sqrt{\beta_{0} q^{\alpha}} \\
-\frac{G r_{0}}{q^{\alpha+1}} \exp -\xi \sqrt{\frac{\operatorname{Pr} q^{\alpha}}{N+1}}, \\
\bar{\theta}(\xi, q)=\frac{1}{q} \exp \left(-\xi \sqrt{\frac{\operatorname{Pr} q^{\alpha}}{N+1}}\right),
\end{array}
$$

with initial and boundary conditions,

$$
\begin{array}{ll}
\bar{V}(\xi, 0)=0, & \bar{\theta}(\xi, 0)=0, \\
\bar{V}(0, q)=\frac{q}{q^{2}+w^{2}}, & \bar{\theta}(0, q)=1, \\
\bar{V}(\infty, q) \rightarrow 0, & \bar{\theta}(\infty, q) \rightarrow 0,
\end{array}
$$


where $G r_{0}=\operatorname{Gr} \beta_{0} /\left(\operatorname{Pr} /(N+1)-\beta_{0}\right)$. Finally, Inverse Laplace equations (11) and (12) will give out the final solution to the problem as follows:

$$
\begin{array}{r}
V(\xi, \tau)=\cos (\omega \tau) \varphi\left(0,-\frac{\alpha}{2},-\xi \sqrt{\frac{\beta_{0}}{\tau^{\alpha}}}\right) \\
+G r_{0} \frac{\tau^{\alpha-1}}{\Gamma(\alpha)}\left[\varphi\left(1,-\frac{\alpha}{2},-\xi \sqrt{\frac{\beta_{0}}{\tau^{\alpha}}}\right)-\varphi\left(1,-\frac{\alpha}{2},-\xi \sqrt{\frac{P r}{(N+1) \tau^{\alpha}}}\right)\right], \\
\theta(\xi, \tau)=\varphi\left(1,-\frac{\alpha}{2},-\xi \sqrt{\frac{P r}{(N+1) \tau^{\alpha}}}\right),
\end{array}
$$

where is the Wright function defined as:

$$
\varphi(a,-\alpha, \tau)=\sum_{n=0}^{\infty} \frac{\tau^{n}}{n ! \Gamma(a-n \alpha)} .
$$

Equations (14) and (15) are bounded by boundary (10).

\subsection{Skin Friction \& Nusselt Number}

Skin friction and Nusselt number in dimensionless form for the solutions from Equations (14) and (15) are as follows:

$$
C_{f}=-\left.\frac{1}{\beta_{0}} \frac{\partial V(\xi, \tau)}{\partial \xi}\right|_{\xi=0},
$$

where is the skin frction and

$$
N u=-\left.\frac{\partial \theta(\xi, \tau)}{\partial \xi}\right|_{\xi=0},
$$

is the Nusslet number.

\section{Results \& Discussions}

Graphical results for velocity and temperature profiles as well numerical results for skin friction and Nusselt number are generated with the aid Mathcad-15 software.

Accuracy of the model and final solution for velocity and temperature profiles is evaluated by comparing the current results with that of Ali et al. [12]. The comparison is shown in Figure 2; as observed, current results are in agreement with published results. To analyse the effects of time, $\tau$ on the velocity of fluid, Figure 3 is plotted. From the figures, it is observed that prolonging time would increase the fluid velocity near the plate. Prolonging time would indicate a longer period for fluid to achieve steady state. To compensate, initial velocity of fluid is usually higher compared to smaller values of $\tau$. As stated in the problem definition, an oscillating plate is considered, over some time velocity of fluid will increase due to boundary condition stated in Equation (10). Figure 4 analyses the influence of Casson fluid parameter, $\beta$ on fluid velocity. It can be seen that by raising $\beta$ values, fluid velocity would decrease. Increasing Casson parameter would cause plasticity and viscosity of the fluid to increase, thus deteriorating the velocity of the fluid. Figure 5 displays profiles for the velocity at different values of $\mathrm{Gr}$. From observation, it can be seen that increment in values would also increase fluid velocity at boundary layer thickness. Increasing Grashof number would increase buoyancy force on fluid. Since fluid is flowing vertically along, an increase in buoyancy force creates an uplift for fluid flow, thus increasing its velocity. It is, however, not the case for $P r$ values. By observing Figure 6, it can be seen that fluid velocity decreases as $\operatorname{Pr}$ values were increased. Thermal diffusivity in fluid will decrease if the Prandtl number is increased, decreasing kinetic energy within fluid, thus decreasing fluid velocity. According to boundary conditions set beforehand in Equation (10), fluid oscillation is observed between -1 and 1 . Figure 7 illustrates the phases of angle, $\omega \tau$. Fluctuations in the angle phase are observed. It is depicted in Figure 8 that fluid velocity increases as thermal radiation, $N$ increases. Kinetic energy withing fluid is increased with an elevated presence of thermal energy, thus increasing velocity of fluid.

Figure 9 illustrates the temperature profile with different values of $\alpha, \operatorname{Pr}$ and $N$. Temperature of fluid decreases as fractional derivative order, $\alpha$ decreases. Increasing $P r$ value would drop the temperature of fluid. Lastly, as the thermal radiation parameter rises, so would the fluid temperature. 


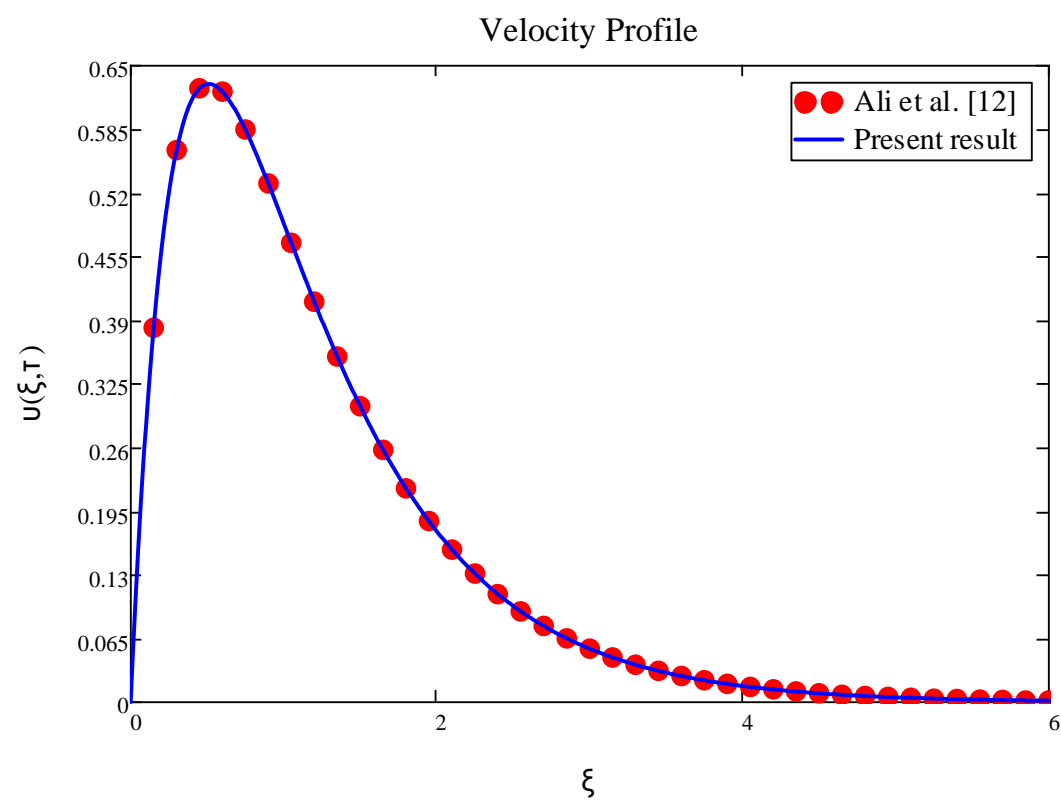

Temperature Profile

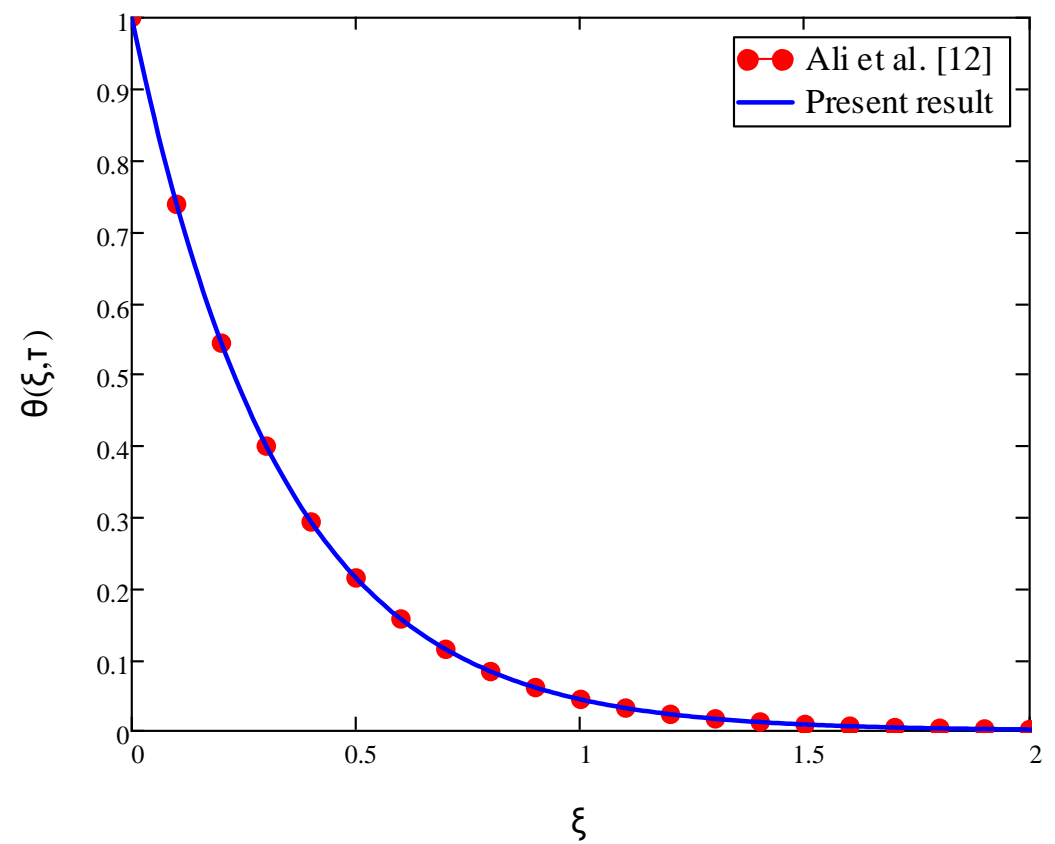

Figure 2. Comparison between Ali et al [12] and present result at $\alpha=0.2, \beta=1, \tau=4, G r=7, \operatorname{Pr}=14, \omega \tau=\pi / 8$ and $N=0$ 

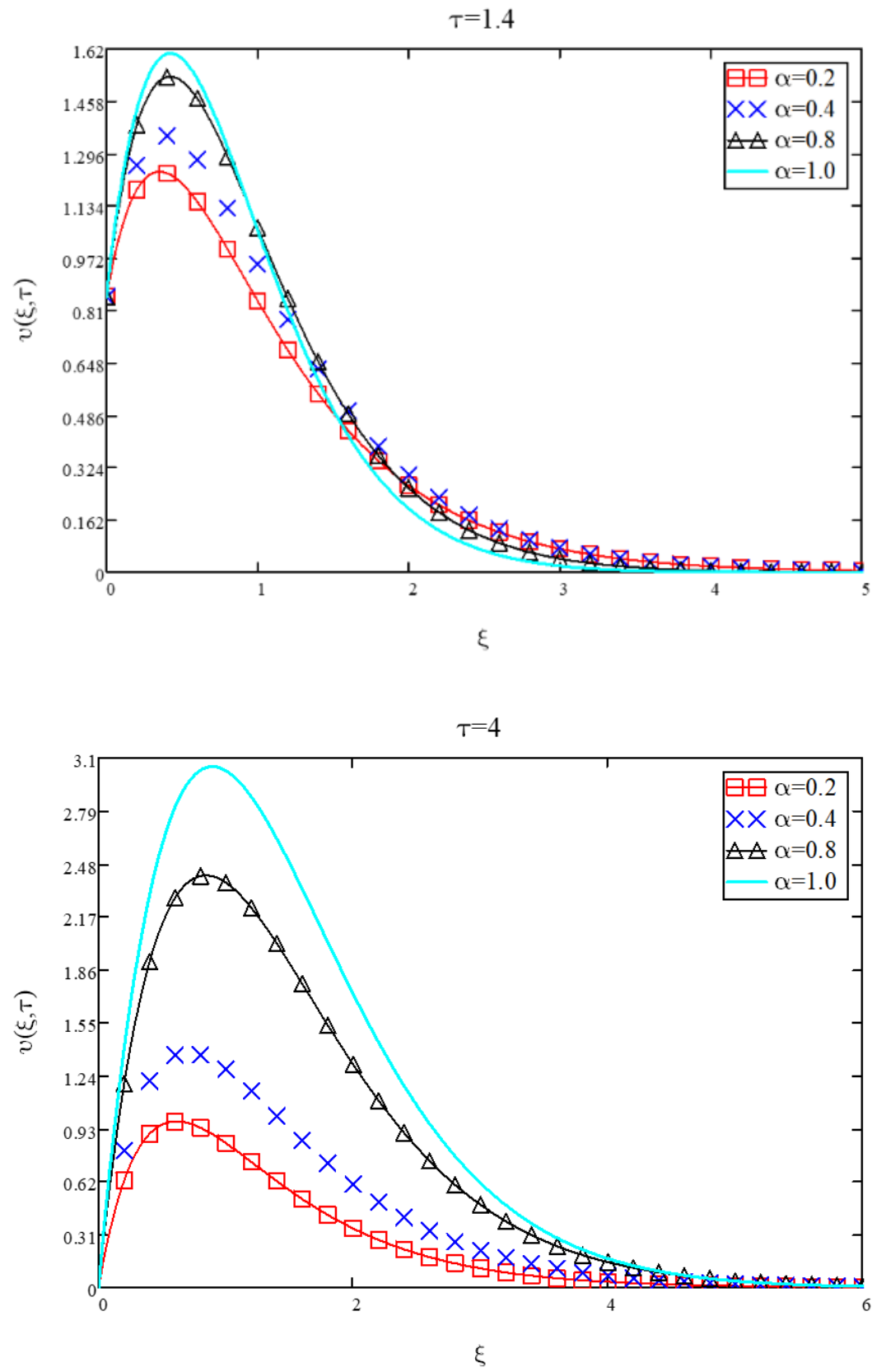

Figure 3. Different values of $\alpha$ and $\tau$ for velocity profile with $\beta=1, G r=7, \operatorname{Pr}=14, \omega \tau=\pi / 8$ and $N=1$ 

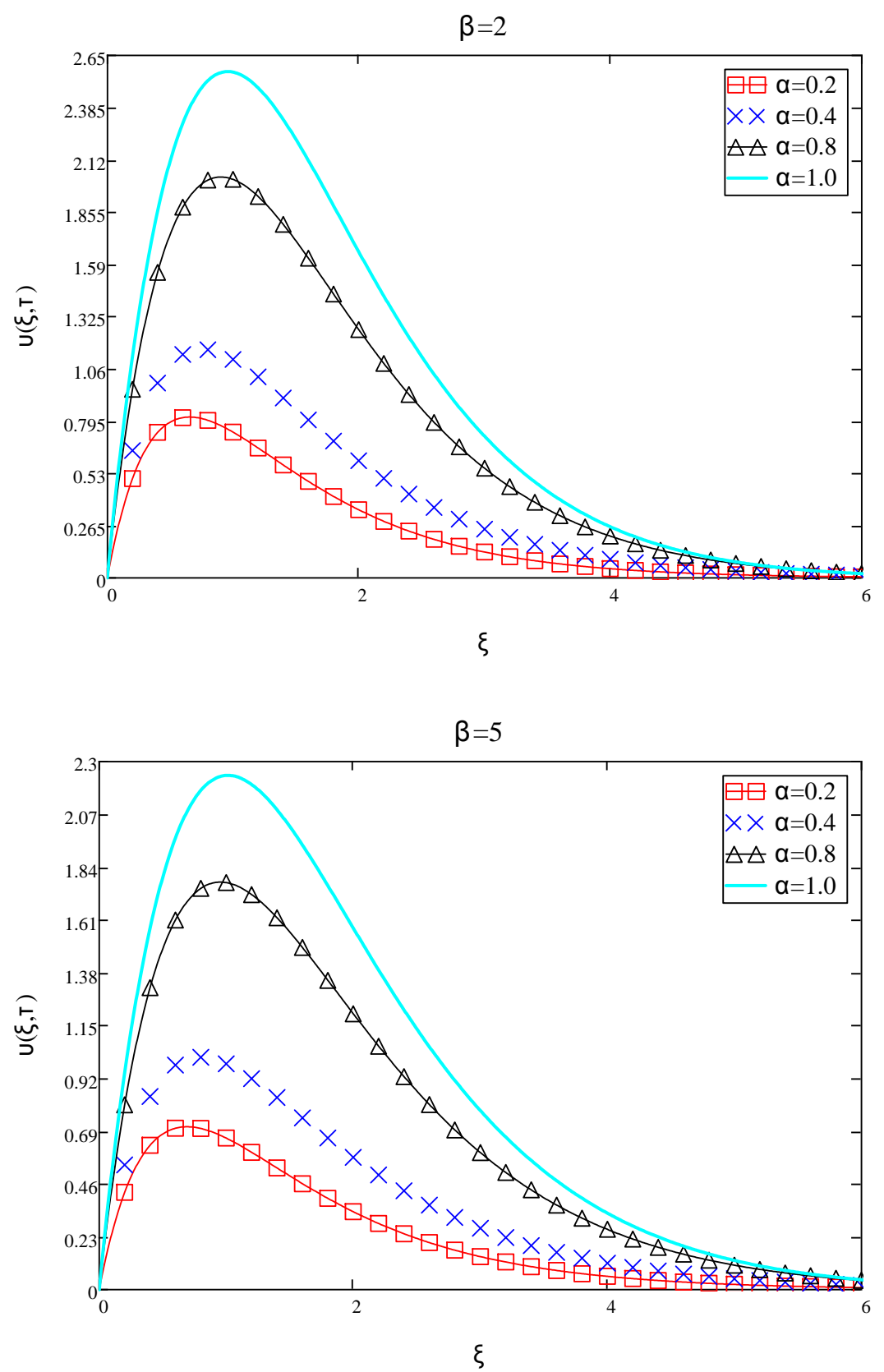

Figure 4. Different values of $\alpha$ and $\beta$ for velocity profile with $\tau=4, G r=7, \operatorname{Pr}=14, \omega \tau=\pi / 8$ and $N=1$ 

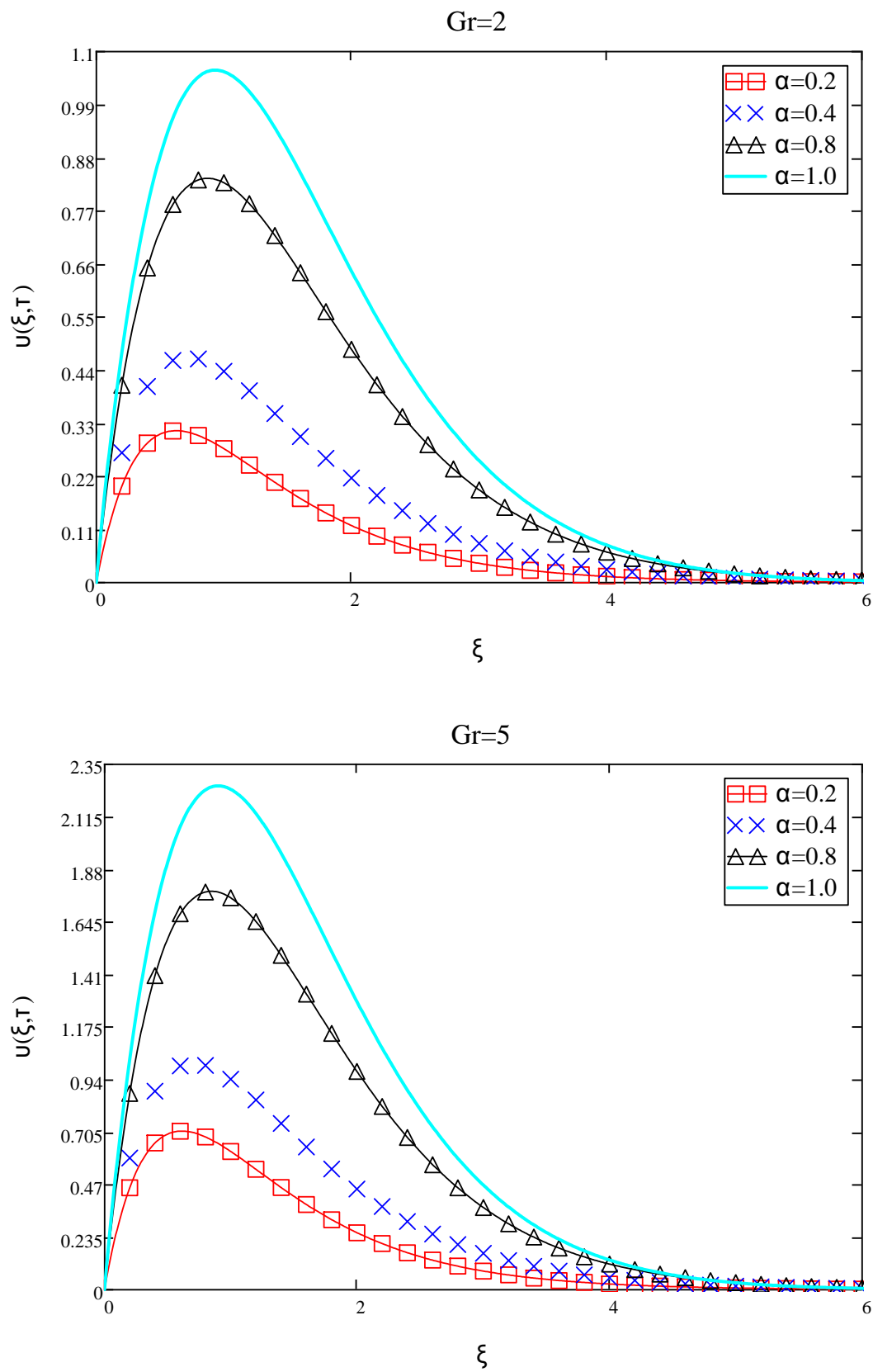

Figure 5. Different values of $\alpha$ and Gr for velocity profile with $\beta=1, \tau=4, \operatorname{Pr}=14, \omega \tau=\pi / 8$ and $N=1$ 

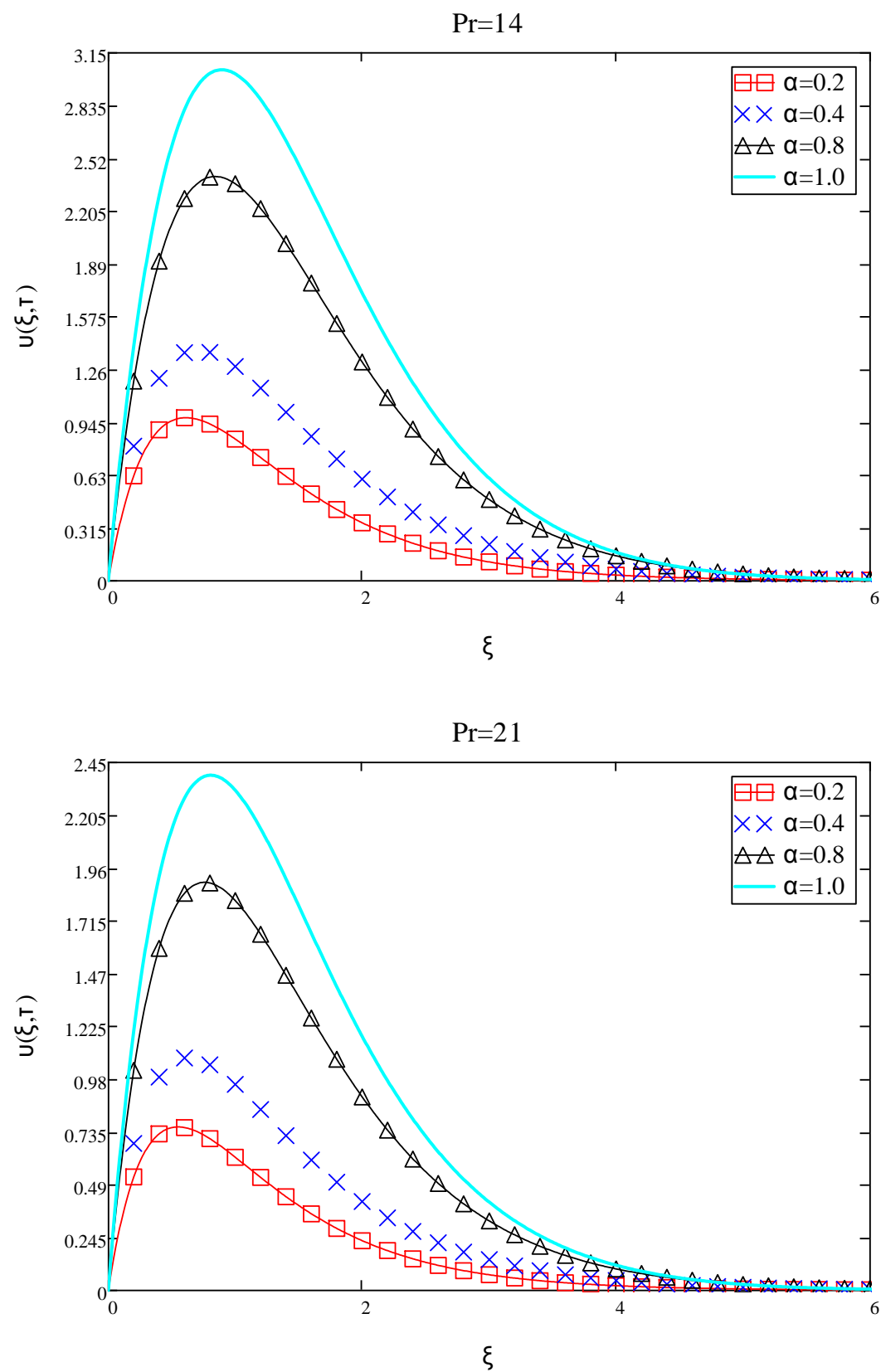

Figure 6. Different values of $\alpha$ and $\operatorname{Pr}$ for velocity profile with $\beta=1, \tau=4, G r=7, \omega \tau=\pi / 8$ and $N=1$ 

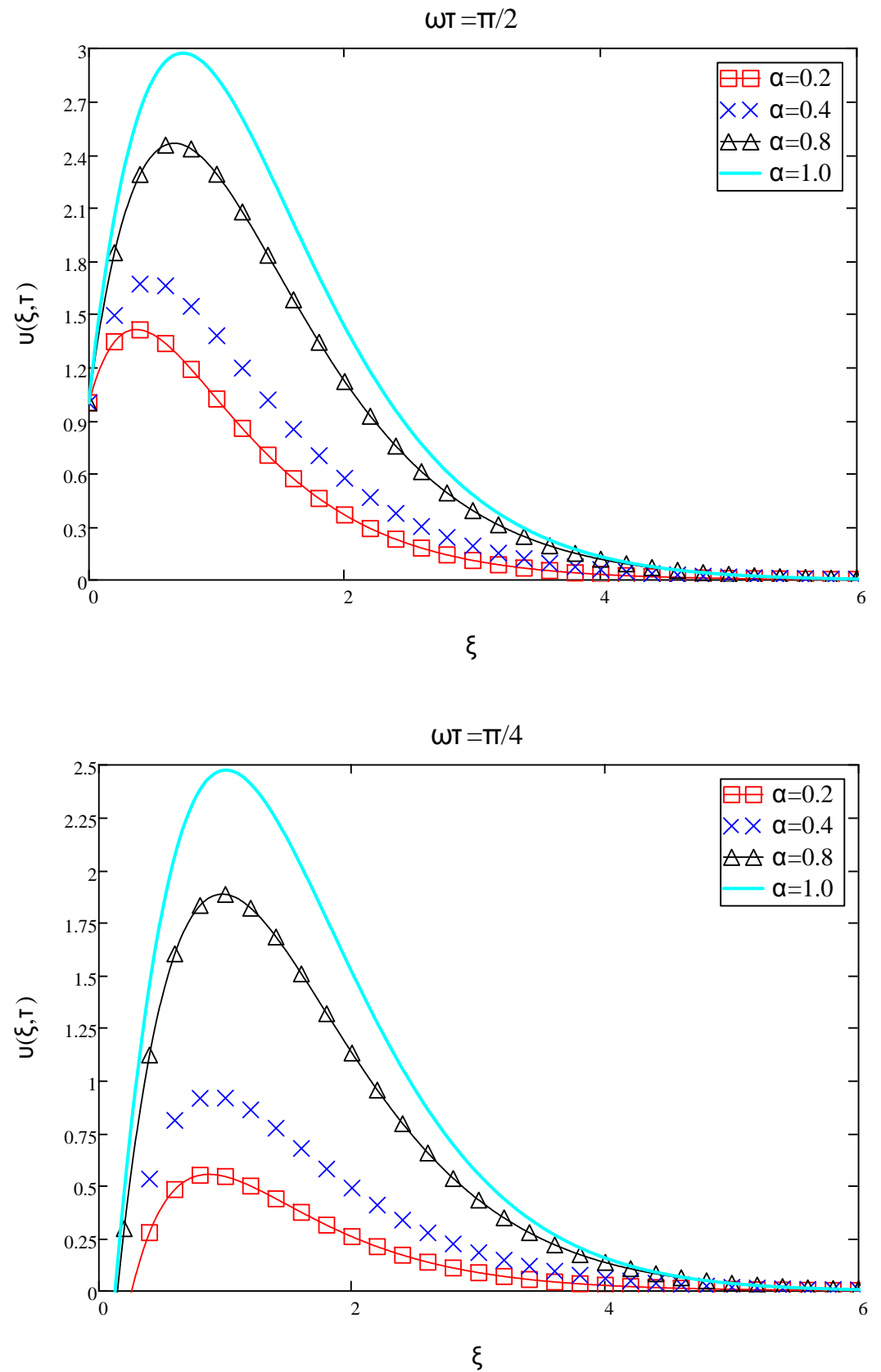

Figure 7. Different values of $\alpha$ and $\omega \tau$ for velocity profile with $\beta=1, \tau=4, G r=7, \operatorname{Pr}=14$ and $N=1$ 

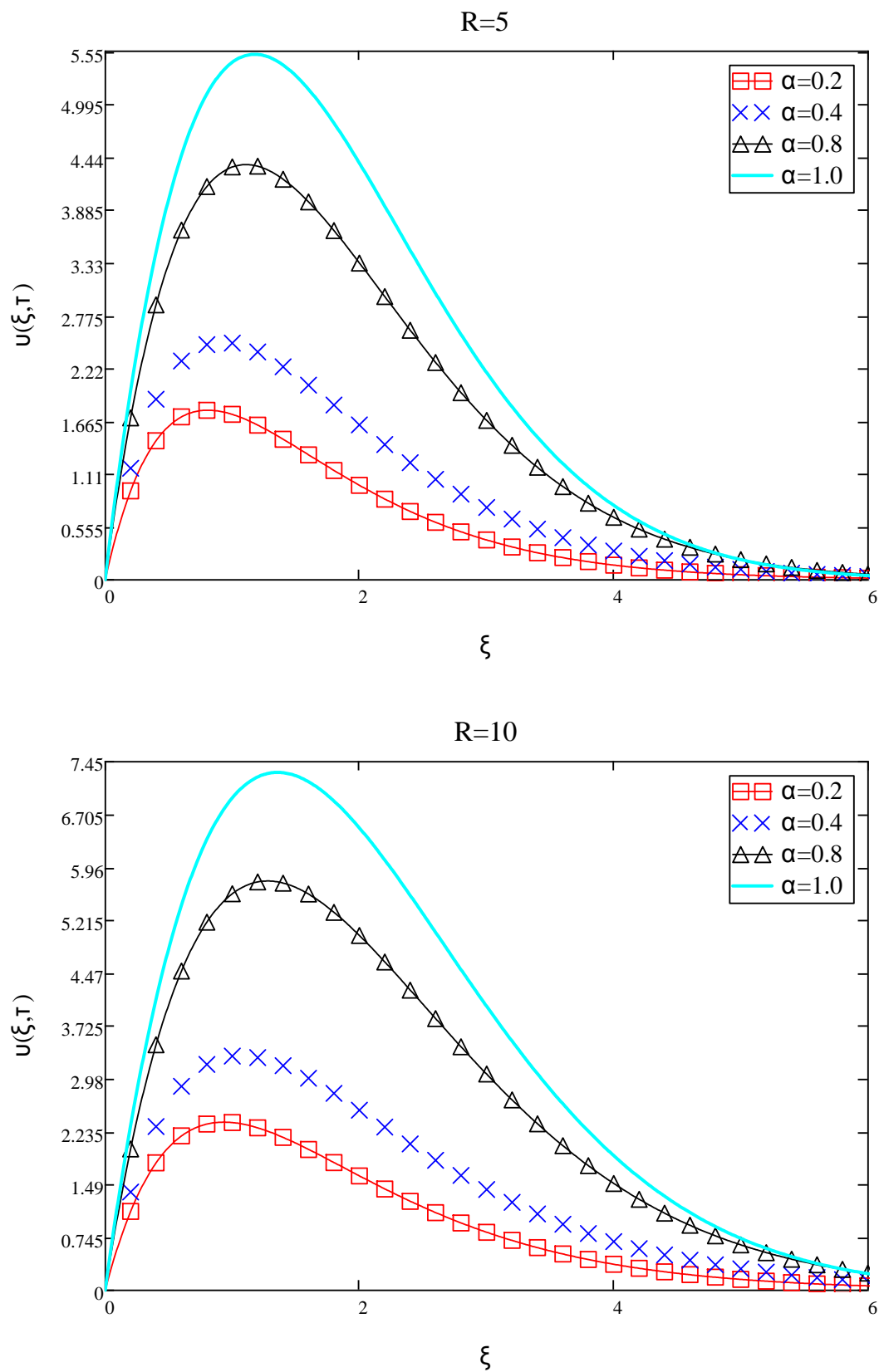

Figure 8. Different values of $\alpha$ and $\mathrm{N}$ for velocity profile with $\beta=1, \tau=4, G r=7, \operatorname{Pr}=14$ and $\omega \tau=\pi / 8$ 

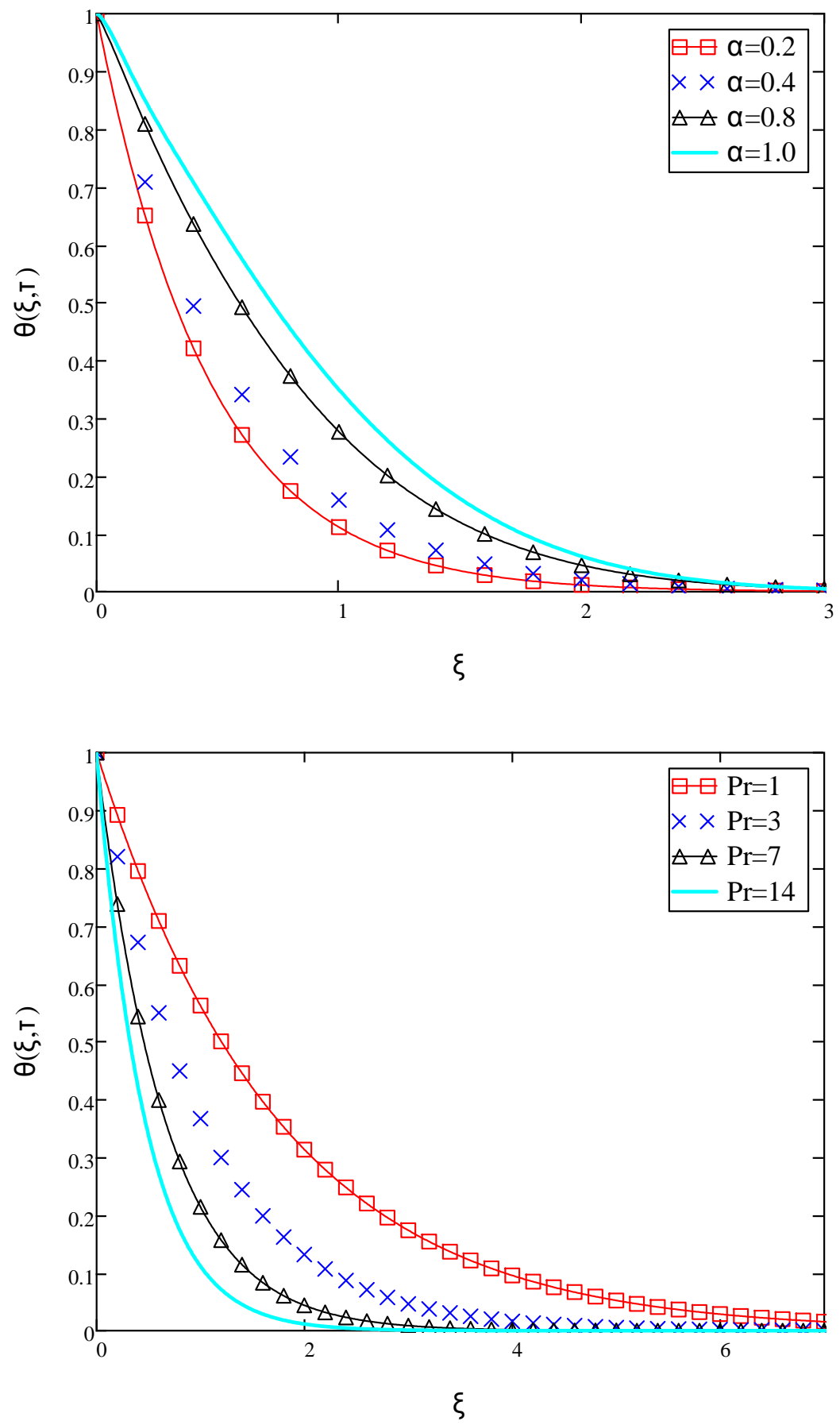


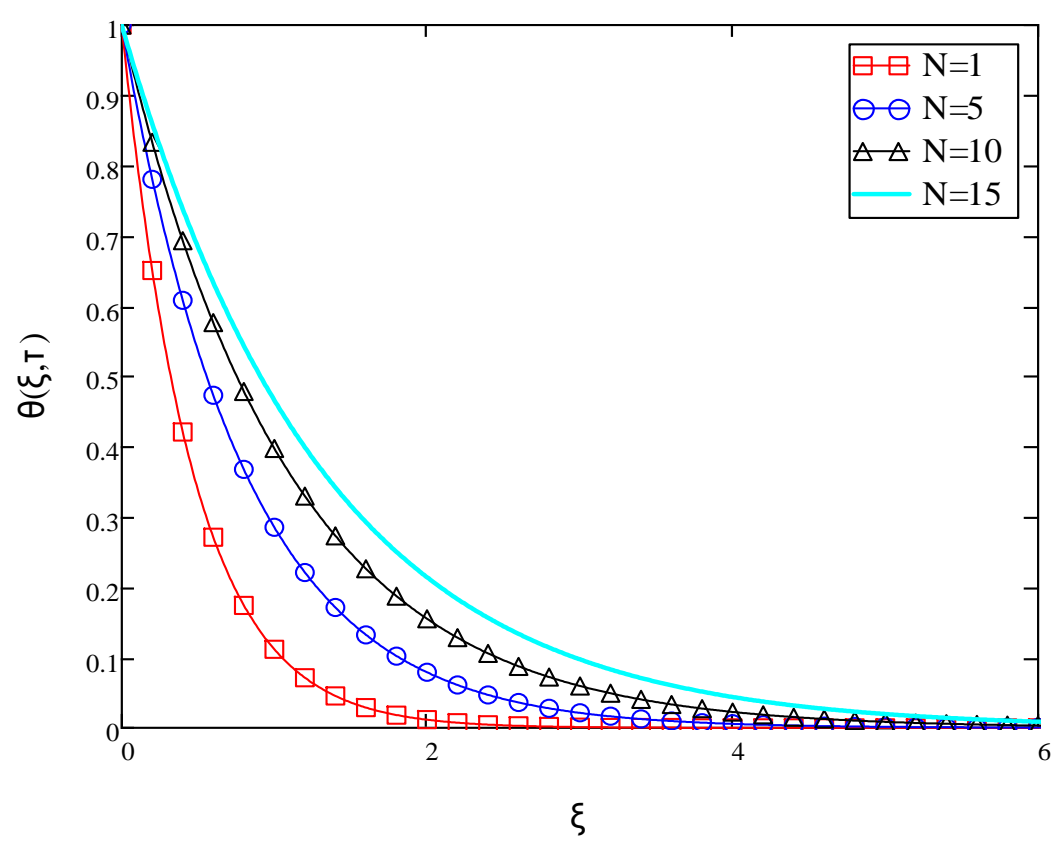

Figure 9. Different values of $\alpha, \operatorname{Pr}$ and $N$ for temperature profile at $\alpha=0.2, \operatorname{Pr}=14$, and $N=1$

Table 1. Nusselt number variations

\begin{tabular}{|c|c|c|c|c|}
\hline $\boldsymbol{\alpha}$ & $\mathbf{P r}$ & $\boldsymbol{\tau}$ & $\boldsymbol{N}$ & $\boldsymbol{N u}$ \\
\hline 0.2 & 7 & 4 & 5 & 0.871 \\
\hline $\mathbf{0 . 8}$ & 7 & 4 & 5 & 0.300 \\
\hline 0.2 & $\mathbf{1 4}$ & 4 & 5 & 1.232 \\
\hline 0.2 & 7 & $\mathbf{8}$ & 5 & 0.813 \\
\hline 0.2 & 7 & 4 & $\mathbf{1 0}$ & 0.644 \\
\hline
\end{tabular}

Table 2. Skin friction variations

\begin{tabular}{|c|c|c|c|c|c|c|c|}
\hline $\boldsymbol{\alpha}$ & $\boldsymbol{\beta}$ & $\boldsymbol{G r}$ & $\mathbf{P r}$ & $\boldsymbol{\tau}$ & $\boldsymbol{\omega} \boldsymbol{\tau}$ & $\boldsymbol{N}$ & $\boldsymbol{C}_{\boldsymbol{f}}$ \\
\hline 0.2 & 0.5 & 7 & 14 & 0.5 & $\pi$ & 5 & 2.206 \\
\hline $\mathbf{0 . 8}$ & 0.5 & 7 & 14 & 0.5 & $\pi$ & 5 & 2.469 \\
\hline 0.2 & $\mathbf{1 . 5}$ & 7 & 14 & 0.5 & $\pi$ & 5 & 2.569 \\
\hline 0.2 & 0.5 & $\mathbf{1 4}$ & 14 & 0.5 & $\pi$ & 5 & 4.318 \\
\hline 0.2 & 0.5 & 7 & $\mathbf{2 1}$ & 0.5 & $\pi$ & 5 & 2.005 \\
\hline 0.2 & 0.5 & 7 & 14 & $\mathbf{1 . 0}$ & $\pi$ & 5 & 2.904 \\
\hline 0.2 & 0.5 & 7 & 14 & 0.5 & $\mathbf{2 \pi}$ & 5 & 2.798 \\
\hline 0.2 & 0.5 & 7 & 14 & 0.5 & $\pi$ & $\mathbf{1 0}$ & 2.501 \\
\hline
\end{tabular}

From Equation (16) and (17), the skin friction and Nusselt number were obtained. Variations of these values were calculated with different values of $\alpha, \operatorname{Pr}, \tau, N, \beta$, $G r$, and $\omega \tau$. As observed, Table 1 displays the variations of Nusselt number, meanwhile Table 2 displays the variations of skin friction. It is observed in Table 1 that by increasing the fractional derivative from 0.2 to 0.8 increases the Nusselt number value. The same phenomenon is observed when increasing values of $\tau$ and $N$. Nusselt number would decrease only by increasing the value of $\mathrm{Pr}$. Nusselt number results correspond well to graphical analysis for temperature profiles displayed in Figure 9. From Table 2, skin friction would increase if the values of $\beta, G r, \tau, \omega \tau$, and $N$. Skin friction would only decrease by increasing the value of $\mathrm{Pr}$. Skin friction results also correspond well to graphical analysis on velocity profile from Figures 2-8.

\section{Conclusions}

This paper aims to develop a fractional derivative model for unsteady boundary layer flow of a Casson fluid past an oscillating vertical plate with constant wall temperature. The dimensionless governing equations are solved using the Laplace transform technique. The results for velocity and temperature are obtained and plotted graphically. The main conclusion of this study are as follows:

I. Both velocity and temperature profiles show increasing behaviour when fractional parameter, $\alpha$, is increased.

II. Velocity increases as values of $\tau, G r$ and $N$ increase. In comparison, as the values of $\beta$ and $\mathrm{Pr}$ increases, the velocity would decrease.

III. The temperature of fluid increases as values of $\alpha$ and $N$ increase and decreases as values of $\mathrm{Pr}$ increases.

IV. Existence of fractional parameter in both velocity and temperature profiles shows the transitional phenomenon of both profiles from an unsteady state to steady state, providing a new perspective on Casson fluid flow. 


\section{Acknowledgements}

The authors would like to acknowledge the Ministry of Higher Education Malaysia and Research Management Centre-UTM, Universiti Teknologi Malaysia (UTM) for financial support through vote numbers FRGS/1/2018/STG06/UTM/02/4，4B583， 02M40 and 08G33.

\section{REFERENCES}

[1] G. W. Leibniz, "Letter from Hanover, Germany, September 30, 1695, to GA L'Hospital," JLeibnizen Math. Schriften, vol. 2 , no. 301-302, p. 1849, 1849.

[2] G. W. Leibniz, "Letter from Hanover, Germany to Johann Bernoulli, December 28, 1695," Leibniz Math. Schriften. Olms-Verlag, Hildesheim, Ger., p. 226, 1962.

[3] G. W. Leibniz, “1697. Letter from Hanover, Germany, to John Wallis, May 28, 1697,” Math. Schriften, 1962.

[4] L. Euler, "De progressionibus transcendentibus seu quarum termini generales algebraice dari nequeunt," Comment. Acad. Sci. Petropolitanae, pp. 36-57, 1738.

[5] P. S. Laplace, Théorie analytique des probabilités. Courcier, 1820.

[6] S. F. Lacroix, "Traité du calcul différentiel et du calcul intégral Tome 3," Trait. du Calc. différentiel du Calc. intégral, 1819.

[7] J. B. J. baron Fourier, Théorie analytique de la chaleur. F. Didot, 1822.

[8] M. Axtell and M. E. Bise, "Fractional calculus application in control systems," in IEEE Conference on Aerospace and Electronics, 1990, pp. 563-566.

[9] S. Das, Functional fractional calculus. Springer Science \& Business Media, 2011.

[10] K. Oldham and J. Spanier, The fractional calculus theory and applications of differentiation and integration to arbitrary order, vol. 111. Elsevier, 1974.

[11] S. G. Samko, A. A. Kilbas, and O. I. Marichev, Fractional integrals and derivatives, vol. 1. Gordon and Breach Science Publishers, Yverdon Yverdon-les-Bains, Switzerland, 1993.

[12] F. Ali, N. A. Sheikh, I. Khan, and M. Saqib, "Solutions with Wright Function for Time Fractional Free Convection Flow of Casson Fluid," Arab. J. Sci. Eng., vol. 42, no. 6, pp. 2565-2572, 2017.

[13] N. A. Sheikh et al., "Comparison and analysis of the Atangana-Baleanu and Caputo-Fabrizio fractional derivatives for generalized Casson fluid model with heat generation and chemical reaction," Results Phys., vol. 7, pp. 789-800, 2017.

[14] L. Debnath, "Recent applications of fractional calculus to science and engineering," Int. J. Math. Math. Sci., vol. 2003, no. 54 , pp. 3413-3442, 2003.

[15] M. Dalir and M. Bashour, "Applications of fractional calculus," Appl. Math. Sci., vol. 4, no. 21-24, pp. 10211032,2010

[16] E. C. De Oliveira and J. A. Tenreiro Machado, "A review of definitions for fractional derivatives and integral," Math. Probl. Eng., vol. 2014, no. 1940, 2014.

[17] E. M. Wright, "On the coefficients of power series having exponential singularities (second paper)," J. London Math. Soc., vol. 1, no. 4, pp. 304-309, 1949.

[18] E. M. Wright, "The generalized Bessel function of order greater than one," Q. J. Math., no. 1, pp. 36-48, 1940.

[19] N. Casson, "A flow equation for pigment-oil suspensions of the printing ink type," Rheol. disperse Syst., 1959.

[20] S. Pramanik, "Casson fluid flow and heat transfer past an exponentially porous stretching surface in presence of thermal radiation," Ain Shams Eng. J., vol. 5, no. 1, pp. 205-212, 2014.

[21] A. Khalid, L. Y. Jiann, I. Khan, and S. Shafie, "Exact solutions for unsteady free convection flow of carbon nanotubes over an oscillating vertical plate," AIP Conf. Proc., vol. 1830, 2017.

[22] I. Khan, N. A. Shah, and D. Vieru, "Unsteady flow of generalized Casson fluid with fractional derivative due to an infinite plate," Eur. Phys. J. Plus, vol. 131, no. 6, p. 181, 2016.

[23] M. Saqib, F. Ali, I. Khan, and N. A. Sheikh, "Heat and mass transfer phenomena in the flow of Casson fluid over an infinite oscillating plate in the presence of first-order chemical reaction and slip effect," Neural Comput. Appl., vol. 30, no. 7, pp. 2159-2172, 2018.

[24] F. Ali, N. A. Sheikh, I. Khan, and M. Saqib, "Magnetic field effect on blood flow of Casson fluid in axisymmetric cylindrical tube: A fractional model," J. Magn. Magn. Mater., vol. 423, no. September 2016, pp. 327-336, 2017.

[25] P. Durga Prasad, R. V. M. S. S. Kiran Kumar, and S. V. K. Varma, "Heat and mass transfer analysis for the MHD flow of nanofluid with radiation absorption," Ain Shams Eng. J., vol. 9, no. 4, pp. 801-813, 2018.

[26] R. Biswas, M. Mondal, D. R. Sarkar, and S. F. Ahmmed, "Effects of Radiation and Chemical Reaction on MHD Unsteady Heat and Mass Transfer of Casson Fluid Flow Past a Vertical Plate," J. Adv. Math. Comput. Sci., vol. 23, no. 2, pp. 1-16, 2017.

[27] A. B. Madhumohana Raju, P. Reddy Chandra, B. Mallikarjuna, and C. S. K. Raju, "RADIATION ABSORPTION AND SORET EFFECTS ON MHD CONDUCTING FLUID FLOW PAST AN EXPONENTIALLY ACCELERATED VERTICAL PLATE," South East Asian J. Math. Math. Sci., vol. 21, no. 1, pp. 1-9, 2020.

[28] M. G. Reddy, P. V. Kumari, and P. Padma, "Effect of thermal radiation on MHD casson nano fluid over a cylinder," J. Nanofluids, vol. 7, no. 3, pp. 428-438, 2018.

[29] S. R. Mohan, "Dufour and Radiation absorption effects on unsteady MHD free convection Casson fluid flow past an 
exponentially infinite vertical plate through porous," vol. 6 no. 2, pp. 485-512, 2019.

[30] F. S. Ibrahim, A. M. Elaiw, and A. A. Bakr, "Effect of the chemical reaction and radiation absorption on the unsteady MHD free convection flow past a semi infinite vertical permeable moving plate with heat source and suction," Commun. Nonlinear Sci. Numer. Simul., vol. 13, no. 6, pp. 1056-1066, 2008.

[31] S. Ramalingeswara Rao, G. Vidyasagar, and G. V. S. R. Deekshitulu, "Unsteady MHD free convection Casson fluid flow past an exponentially accelerated infinite vertical porous plate through porous medium in the presence of radiation absorption with heat generation/absorption," Mater. Today Proc., no. xxxx, 2020.

[32] R. Mahato and M. Das, "Effect of suction/blowing on heat-absorbing unsteady radiative Casson fluid past a semi-infinite flat plate with conjugate heating and inclined magnetic field," Pramana - J. Phys., vol. 94, no. 1, pp. 1-16, 2020.

[33] M. T. Akolade, A. T. Adeosun, and J. O. Olabode, "Influence of Thermophysical Features on MHD Squeezed Flow of Dissipative Casson fluid with Chemical and Radiative Effects," J. Appl. Comput. Mech., no. x, pp. 1-11, 2020.

[34] Z. Abbas, M. Sheikh, and S. S. Motsa, "Numerical solution of binary chemical reaction on stagnation point flow of Casson fluid over a stretching/shrinking sheet with thermal radiation," Energy, vol. 95, pp. 12-20, 2016.
[35] C. Fetecau, D. Vieru, and W. A. Azhar, "Natural convection flow of fractional nanofluids over an isothermal vertical plate with thermal radiation," Appl. Sci., vol. 7, no. 3, 2017.

[36] H. R. Kataria and H. R. Patel, "Radiation and chemical reaction effects on MHD Casson fluid flow past an oscillating vertical plate embedded in porous medium," Alexandria Eng. J., vol. 55, no. 1, pp. 583-595, 2016.

[37] M. Najib Zakaria, A. Hussanan, I. Khan, and S. Shafie, "The effects of radiation on free convection flow with ramped wall temperature in brinkman type fluid," J. Teknol. (Sciences Eng., vol. 62, no. 3, pp. 33-39, 2013.

[38] S. Maiti, S. Shaw, and G. C. Shit, "Caputo-Fabrizio fractional order model on MHD blood flow with heat and mass transfer through a porous vessel in the presence of thermal radiation," Phys. A Stat. Mech. its Appl., vol. 540, p. 123149, 2020.

[39] N. Sene, "A new approach for the solutions of the fractional generalized Casson uid model described by Caputo fractional operator," Adv. Theory Nonlinear Anal. its Appl., vol. 4, no. 4, pp. 373-384, 2020.

[40] N. Sene, "Research Article A Numerical Algorithm Applied to Free Convection Flows of the Casson Fluid along with Heat and Mass Transfer Described by the Caputo Derivative," Adv. Math. Phys., vol. 2021, 2021.

[41] A. Tassaddiq, I. Khan, K. Sooppy, and J. Singh, "MHD flow of a generalized Casson fluid with Newtonian heating: A fractional model with Mittag - Leffler memory," Alexandria Eng. J., vol. 59, no. 5, pp. 3049-3059, 2020. 